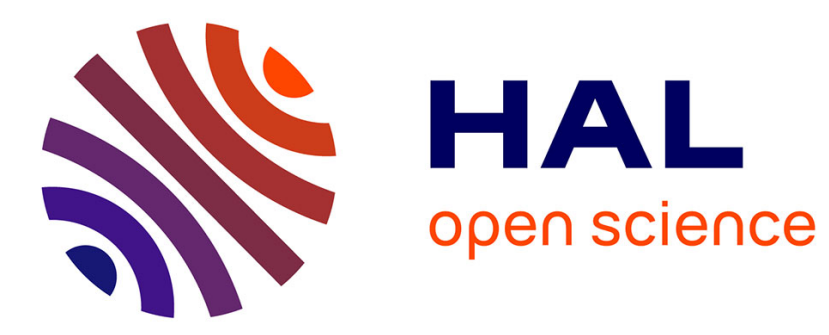

\title{
Subspace-based damage detection on steel frame structure under changing excitation
}

\author{
Michael Döhler, Falk Hille
}

\section{To cite this version:}

Michael Döhler, Falk Hille. Subspace-based damage detection on steel frame structure under changing excitation. IMAC - 32nd International Modal Analysis Conference, SEM, Feb 2014, Orlando, United States. 10.1007/978-3-319-04570-2_19. hal-00953131

\section{HAL Id: hal-00953131 \\ https://hal.inria.fr/hal-00953131}

Submitted on 28 Feb 2014

HAL is a multi-disciplinary open access archive for the deposit and dissemination of scientific research documents, whether they are published or not. The documents may come from teaching and research institutions in France or abroad, or from public or private research centers.
L'archive ouverte pluridisciplinaire HAL, est destinée au dépôt et à la diffusion de documents scientifiques de niveau recherche, publiés ou non, émanant des établissements d'enseignement et de recherche français ou étrangers, des laboratoires publics ou privés. 


\title{
Subspace-based damage detection on steel frame structure under changing excitation
}

\author{
M. Döhler ${ }^{1,2}$ and F. Hille ${ }^{1}$ \\ ${ }^{1}$ BAM Federal Institute for Materials Research and Testing, Safety of Structures Department, 12200 Berlin, Germany \\ ${ }^{2}$ Inria, Campus de Beaulieu, 35042 Rennes, France
}

\begin{abstract}
Damage detection can be performed by detecting changes in the modal parameters between a reference state and the current (possibly damaged) state of a structure from measured output-only vibration data. Alternatively, a subspace-based damage detection test has been proposed and applied successfully, where changes in the modal parameters are detected, but the estimation of the modal parameters themselves is avoided. Like this, the test can run in an automated way directly on the vibration measurements. However, it was assumed that the unmeasured ambient excitation properties during measurements of the structure in the reference and possibly damaged condition stay constant, which is hardly satisfied by any application. A new version of the test has been derived recently that is robust to such changes in the ambient excitation. In this paper, the robust test is recalled and its performance is evaluated both on numerical simulations and a real application, where a steel frame structure is artificially damaged in the lab.
\end{abstract}

Keywords: Damage detection; Ambient vibration; Changing excitation; Subspace methods; Robust tests.

\section{Introduction}

In the last twenty years, monitoring the integrity of the civil infrastructure has been an active research topic, including in connected areas such as automatic control, for mastering the aging of bridges, or the resistance to seismic events and the protection of the cultural heritage. The structural response to unknown ambient excitation is measured, and vibration-based monitoring can be performed under the premise that changes in the structural properties due to damage lead to changes in the eigenstructure of a system. Since the ambient excitation is unknown, it can change during the monitoring of a structure, e.g. in changing wind or traffic conditions. The focus of this paper is damage detection under possible changes in the ambient excitation.

A standard approach for damage detection is modal parameter identification and their comparison to a reference state of the monitored structure, e.g. as in $[8,11]$. The modal parameters are not afflicted by different ambient excitations, but their automatic estimation and matching from measurements of different states of the structure might require an extensive preprocessing step.

In this paper we consider the subspace-based damage detection approach developed in $[2,3,5,6]$. With this approach, a data-driven model obtained in the reference state is compared to data from the possibly damaged state using a subspacebased residual function and a $\chi^{2}$-test built on it for a hypothesis test, without actually estimating the modal parameters in the tested, possibly damaged states. This has the advantage that the computations in the tested (possibly damaged) state are fully automated. The computed $\chi^{2}$-value is compared to a threshold to decide if damage occurred or not.

Since this kind of methods process the measurement data in a much more direct way, the original algorithms from [2, 3] have the drawback of being influenced by changes in the unknown ambient excitation, which may lead to false alarms. Recently, the original algorithms have been extended and modified to be robust under such changes in the excitation in [6]. This approach has been shown to be promising under changing excitation in numerical simulations $[7,12,6]$. It is the purpose of this paper to validate the new approach on a case study in the lab under different excitation levels.

This paper is organized as follows. In Section 2, the basics of the considered damage detection approach are presented and in Section 3 the extension from [6] that is robust to changing ambient excitation. In Section 4, a numerical application is presented on a simulated truss, as well as a case study on a steel frame structure in the lab that was artificially damaged. Finally, concluding remarks are given in Section 5. 


\section{Subspace-based damage detection}

\subsection{Models and parameters}

The behavior of a structure is assumed to be described by a linear time-invariant (LTI) dynamical system

$$
\mathcal{M} \ddot{X}(t)+C \dot{X}(t)+\mathcal{K} X(t)=v(t)
$$

where $t$ denotes continuous time, $\mathcal{M}, \mathcal{C}, \mathcal{K} \in \mathbb{R}^{d \times d}$ are the mass, damping and stiffness matrices respectively and $X \in \mathbb{R}^{d}$ collects the displacements of the $d$ degrees of freedom (DOF) of the structure. The external and non-measured force $v(t)$ is modeled as white noise.

Let the system (1) be observed at $r$ coordinates, e.g. with accelerometers. Discretizing system (1) in time and transformation to a first order system leads to the discrete time state-space model

$$
\left\{\begin{aligned}
x_{k+1} & =A x_{k}+v_{k} \\
y_{k} & =C x_{k}+w_{k}
\end{aligned}\right.
$$

with the states $x_{k} \in \mathbb{R}^{n}$, the outputs $y_{k} \in \mathbb{R}^{r}$, the state transition matrix $A \in \mathbb{R}^{n \times n}$ and the observation matrix $C \in \mathbb{R}^{r \times n}$, where $r$ is the number of sensors and $n$ is the system order. The excitation $v_{k}$ is an unmeasured Gaussian white noise sequence with zero mean and (during a measurement) constant covariance matrix $Q: \mathbf{E}\left(v_{k} v_{k^{\prime}}^{T}\right) \stackrel{\text { def }}{=} Q \delta\left(k-k^{\prime}\right)$, where $\mathbf{E}(\cdot)$ denotes the expectation operator, and $w_{k}$ is the measurement noise.

\subsection{Properties from subspace-based system identification}

For the damage detection method in [2, 3] a residual function is constructed based on properties from covariance-driven outputonly subspace-based system identification $[13,10]$.

Let $G=\mathbf{E}\left(x_{k+1} y_{k}^{T}\right)$ be the cross-covariance between the states and the outputs, let $R_{i}=\mathbf{E}\left(y_{k} y_{k-i}^{T}\right)=C A^{i-1} G$ be the theoretic output covariances and

$$
\mathcal{H}_{p+1, q} \stackrel{\text { def }}{=}\left[\begin{array}{cccc}
R_{1} & R_{2} & \ldots & R_{q} \\
R_{2} & R_{3} & \ldots & R_{q+1} \\
\vdots & \vdots & \ddots & \vdots \\
R_{p+1} & R_{p+2} & \ldots & R_{p+q}
\end{array}\right] \stackrel{\text { def }}{=} \operatorname{Hank}\left(R_{i}\right)
$$

be the theoretic block Hankel matrix of size $(p+1) r \times q r$, where parameters $p$ and $q$ are chosen such that $\min \{p r, q r\} \geq n$ with often $p+1=q$. Matrix $\mathcal{H}_{p+1, q}$ possesses the well-known factorization property

$$
\mathcal{H}_{p+1, q}=O_{p+1} \mathcal{C}_{q}
$$

into the matrices of observability and controllability

$$
O_{p+1}=\left[\begin{array}{c}
C \\
C A \\
\vdots \\
C A^{p}
\end{array}\right], \quad C_{q}=\left[\begin{array}{llll}
G & A G & \ldots & A^{q-1} G
\end{array}\right] .
$$

From the observability matrix $O_{p+1}$, the matrices $C$ and $A$ could be recovered $[13,10]$ and subsequently the modal parameters. However, the fact is used that damages lead to changes in $A$ and $C$ and subsequently in $\mathcal{H}_{p+1, q}$ through properties 4 and 5 , which will be directly checked in a statistical test, instead of doing system identification.

\subsection{The damage detection test}

In the following, the non-parametric damage detection test [1] based on [2, 3] is described. Using measured data $\left(y_{k}\right)_{k=1, \ldots, N}$, a consistent estimate $\widehat{\mathcal{H}}_{p+1, q}$ of the Hankel matrix is obtained from the empirical output covariances

$$
\widehat{R}_{i}=\frac{1}{N} \sum_{k=1}^{N} y_{k} y_{k-i}^{T}, \quad \widehat{\mathcal{H}}_{p+1, q}=\operatorname{Hank}\left(\widehat{R}_{i}\right) .
$$


Let $\widehat{\mathcal{H}}_{p+1, q}^{\text {ref }}$ be the Hankel matrix in the reference state. Compute its left null space $S$ from the singular value decomposition (SVD)

$$
\widehat{\mathcal{H}}_{p+1, q}^{\mathrm{ref}}=\left[\begin{array}{ll}
\widehat{U}_{1} & \widehat{U}_{0}
\end{array}\right]\left[\begin{array}{cc}
\widehat{\Delta}_{1} & 0 \\
0 & \widehat{\Delta}_{0}
\end{array}\right]\left[\begin{array}{c}
\widehat{V}_{1}^{T} \\
\widehat{V}_{0}^{T}
\end{array}\right]
$$

as $S=\widehat{U}_{0}$, where $\widehat{\Delta}_{1}$ is of size $n \times n$ and where $\widehat{\Delta}_{0} \approx 0$.

The characteristic property of the reference state then writes as

$$
S^{T} \widehat{\mathcal{H}}_{p+1, q} \approx 0
$$

while the product deviates from 0 in the damaged state. To decide whether measured data corresponds to the reference state or not, the residual vector $\zeta$ with

$$
\zeta=\sqrt{N} \operatorname{vec}\left(S^{T} \widehat{\mathcal{H}}_{p+1, q}\right)
$$

is defined [2, 3]. It is tested if this residual is significantly different from zero or not, using the $\chi^{2}$ test

$$
\chi_{\zeta}^{2}=\zeta^{T} \Sigma_{\zeta}^{-1} \zeta
$$

where the empirical residual covariance $\Sigma_{\zeta}=\operatorname{cov}(\zeta)$ is computed on several datasets from the reference state. To decide if damage occurred or not, the test value $\chi^{2}$ is compared to a threshold, which can also obtained from $\chi^{2}$ test values on several datasets from the reference state.

\section{Robust damage detection test under changing excitation}

A change in the covariance $Q$ of the unmeasured ambient excitation $v_{k}$ of system (2) provokes a change in the the crosscovariance $G$ between the states and the outputs and thus in the Hankel matrix estimate $\widehat{\mathcal{H}}_{p+1, q}$ (see (3)), even if no structural change occurs. Hence, the residual $\zeta$ and the corresponding test value $\chi^{2}$ are influenced by changes in the ambient excitation, which may lead to false alarms.

Due to these reasons, a robust variant of the presented damage detection test was derived in [6]. It is based on the property that the Hankel matrix $\widehat{\mathcal{H}}_{p+1, q}$ and the matrix of its principal left singular vectors $\widehat{U}_{1}$ (see SVD in (7)) share the same left null space $S$ - another characteristic property of the reference state can be written as

$$
S^{T} \widehat{U}_{1} \approx 0
$$

analogous to (8). While the Hankel matrix $\widehat{\mathcal{H}}_{p+1, q}$ is dependent on the ambient excitation properties, matrix $\widehat{U}_{1}$ is a matrix with orthonormal columns and thus can be regarded as independent of the excitation properties. Note that matrix $\widehat{U}_{1}$ is defined by a unique SVD (e.g. by forcing the first entry in each column to be positive) to ensure no changing modal basis.

Since $\widehat{U}_{1}$ is independent of the excitation, a robust residual vector is defined as

$$
\xi=\sqrt{N} \operatorname{vec}\left(S^{T} \widehat{U}_{1}\right)
$$

is defined [6]. It is tested if this residual is significantly different from zero or not, using the $\chi^{2}$ test

$$
\chi_{\xi}^{2}=\xi^{T} \Sigma_{\xi}^{-1} \xi
$$

where the empirical residual covariance $\Sigma_{\xi}=\operatorname{cov}(\xi)$ is computed on several datasets from the reference state as detailed in [6].

\section{Applications}

\subsection{Numerical application: simulated truss structure}

A truss model with 25 DOF (see Figure 1) was considered in a first numerical application as in [6]. The output data was generated at six sensor positions in vertical direction at the lower chord by exciting the structure at the same positions with white noise. The excitation noise at these six positions was generated with a diagonal covariance matrix $Q$, whose diagonal entries were randomly chosen from a uniform distribution in the interval $[1,36] .5 \%$ white noise were added on the generated outputs. 


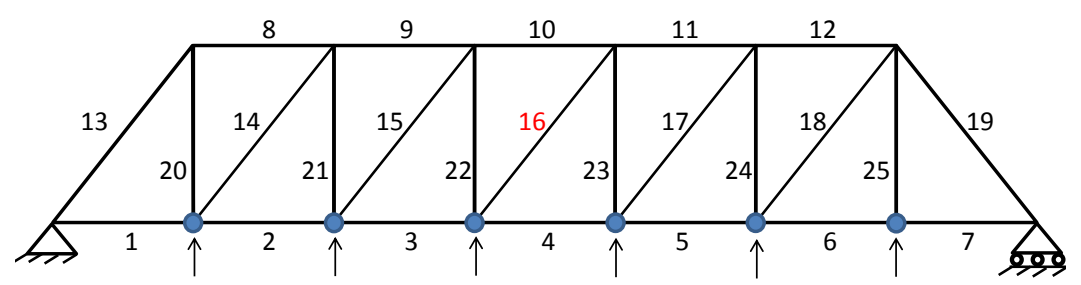

Figure 1: Truss structure with six sensors.

Damage was simulated by decreasing the stiffness of element 16 in a first step by $10 \%$ and in a second step by $20 \%$, leading to a decrease of the structure's natural frequencies of up to $1.0 \%$ and $2.2 \%$ compared to the reference state, respectively. In the reference state, the null space $S$ and the residual covariance $\Sigma_{\zeta}$ (for the conventional test (10)) and $\Sigma_{\xi}$ (for the robust test (12)) were computed on 10 data sets to set up the parameters of the damage detection tests.

In each structural state, data sets of length $N=25000$ at a sampling frequency of $50 \mathrm{~Hz}$ were generated. To compare both damage detection tests under changing excitation, 100 new data samples were generated with random excitation covariance in the reference and both damaged states, on which the $\chi^{2}$-tests were computed in Figure 2 . For each of the compared tests, an empirical threshold was computed from the $\chi^{2}$-values of the reference state allowing a $5 \%$ type I error (horizontal dashed line).

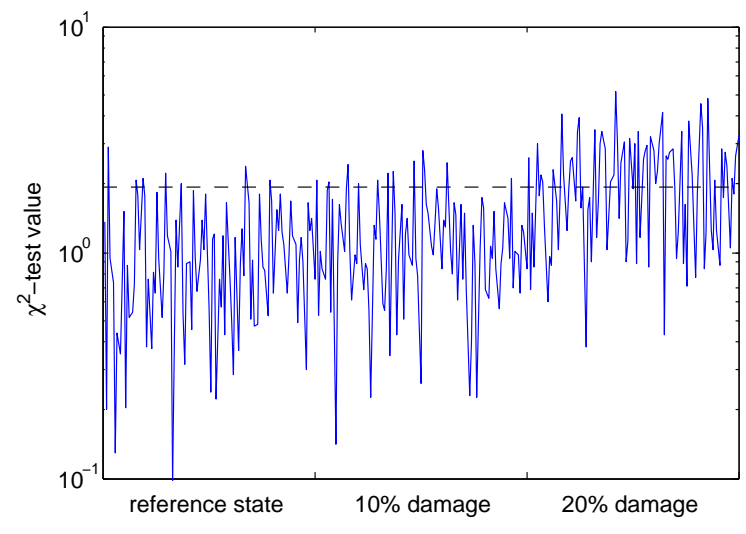

(a) Conventional test (10).



(b) New robust test (12).

Figure 2: Comparison of damage detection tests on simulated truss structure (log-scale).

As can be seen in Figure 2(a), the damage detection fails with the conventional test under changing excitation properties, while the new robust damage detection test in Figure 2(b) manages to separate the reference state from the damaged states very well.

\subsection{Laboratory application: Steel frame structure}

A test series has been performed at a jacket-like steel structures within the lab facilities of BAM to verify the usability, reliability and robustness of the introduced damage detection algorithm under changing excitation properties. The test structure provides the ability to gradually and reversibly increase artificially induced structural damage. The test stand can be used to analyze vibration based damage identification methods or just the sensitivity of single parameters influencing the identification of structural damage.

\subsubsection{The lab structure and damage modeling}

For the lack of options to test damage identification methods on real size civil engineering structures it is common to experimentally analyze newly developed procedures on scaled medium size laboratory structures. This is especially true for new structural types as e.g. offshore wind energy converters. As part of the research work on subspace-based damage detection on offshore structures at BAM a particular steel frame structure has been constructed and is provided as laboratory model. 
The model structure is made of steel pipe components representing a scaled two-dimensional section of a jacket-type support structure for an offshore wind energy converter. For this purpose one and a half diagonal bracings are designed between the two legs. The upper completion is formed by an I-sectional steel beam. At the lower end the legs end in steel foot plates. With exception of the damage sections all structural parts of the model are welded. The foot plates are screwed via a transition piece to the floor. In the z-direction the structure is held on its head girder by a complex construction, whose two fork-like support poles allow a movement of the structure in the x-direction.


Figure 3: Test stand for proof testing of damage identification algorithms; left: laboratory structure, middle: flange connection for damage modeling, right: electrodynamic shaker for excitation

The lab structure is constructed such that a defined local loss of stiffness can be modeled, which complies with the requirements of a realistic projection of an individual fatigue crack as a damage, and the reversibility of the induced damages is assured for the repeatability of test series. Though, cracks as damage can be induced artificially at one K-type gusset of the model structure by loosening bolts of a flange connection. Four of such flange connections were designed, each at the four ends of the $\mathrm{K}$-type gusset. The progress of a crack-like damage is then simulated by the number of bolts that are loosened. Each flange joint was equipped with a maximal possible number of bolts to provide a high resolution of the induced stiffness loss.

\subsubsection{Measurement setup}

The excitation for the tests was provided by an electrodynamic shaker. A broadband random acceleration signal with a frequency content between $10 \mathrm{~Hz}$ and $1000 \mathrm{~Hz}$ was produced by a shaker control unit and was induced via the shaker at the top of the structure after amplification. The shaker was equipped with a $5 \mathrm{~kg}$ excitation mass and the excitation direction was horizontal and approximately $30^{\circ}$ rotated out of the in-plane-direction.

Nine piezoelectric accelerometers were applied at the structure for the vibration measurements. The sensors were connected with the structure by permanent magnets and measured in the direction perpendicular to the surface they were applied to. The signals were amplified, recorded and stored with a 20 channel DIFA measurement unit.

\subsubsection{Damage detection test}

A test series was conducted to validate the reliability and sensitivity of the described robust damage detection procedure under changing excitation. In the tests, the time signals were measured for $16.4 \mathrm{~s}$ with a sampling rate of $2500 \mathrm{~Hz}$, which results in 40,960 data points per signal.

The damage location was chosen at the lower brace of the flange. In the reference state all bolts were screwed tight. For inducing damage, successively 1, 2, 3, 5 or 7 adjacent bolts were unscrewed, each representing a fatigue crack of increasing length. Thereby, the loosening of 3 bolts is comparable with a reduction of the moment of inertia by $3 \%$, whereas the unscrewing of 7 bolts means a loss of already $30 \%$ of the bending stiffness.

To validate the damage detection algorithm under changing excitation, three excitation (power) levels were used for each damage state. Besides the full scale level, a reduction of $5 \mathrm{~dB}$ and a reduction of $10 \mathrm{~dB}$ with respect to the full scale level were performed and are denoted as "Excitation 1", "Excitation 2" and "Excitation 3" in the tests, respectively. A reduction of $5 \mathrm{~dB}$ 
accounts for a power ratio of $\approx 0.31$ and an amplitude ratio of $\approx 0.56$ and a reduction of $10 \mathrm{~dB}$ for a power ratio of 0.1 and an amplitude ratio of $\approx 0.31$. The full scale excitation power level was chosen preliminary in dependence of the shaker performance. For each damage level and each excitation level, four signals of $16.4 \mathrm{~s}$ length was measured.

\subsubsection{Results}

Both the conventional damage detection test presented in Section 2.3 and the robust damage detection test under changing excitation in Section 3 are applied to the measured data with exactly the same parameters.

Two data sets from Excitation 1 and two data sets from Excitation 3 in the reference state are used to compute the null space $S$ and the covariance $\Sigma$ for both test variants. The tests were set up with the parameters of the block Hankel matrix $p+1=q=15$ and model order $n=120$, resulting in the dimension of the null space $S$ of $q r-n=15$. The mentioned data sets were separated into altogether 1000 pieces for the computation of the covariance matrix. See also reference [6] for the numerical details and an efficient implementation of these computations.

Then, both tests (see Equations (10) and (12), respectively) are applied to all the measured data sets in the reference and damaged states at the three different excitation levels. The computed $\chi^{2}$ test values are presented in Figures 4(a) and 4(b) for both tests, respectively, together with an empirical threshold that was set up using the $\chi^{2}$ test values of the reference state.

In Figure 4(a) it can be seen that the conventional test reacts strongly to different excitation levels. A decrease in the excitation level results also in a decrease in the $\chi^{2}$ test value, independently of the damage. While the $\chi^{2}$ test values increase with the damage size for each excitation level, this increase is not distinguishable for the different excitation levels. Only the $\chi^{2}$ test values of a significant damage (7 loosened bolts) all lie over the threshold that was established in the reference state. Thus, only damages can be clearly detected with the conventional test, whose influence on the $\chi^{2}$ test is stronger than the changes in excitation.

On the other side, the results of the new test in Figure 4(b) clearly show a stronger robustness to changing excitation properties. While there are still small fluctuations of the test values in the reference state and in the states with small damage, these fluctuations are less significant. For the damages from 3 loosened bolts on, these variations seem to be independent from the excitation level, which validates the robustness of the new test to changing excitation. Furthermore, the relatively small damage of only 3 loosened bolts can be clearly detected under changing excitation with the new test thanks to its robustness.

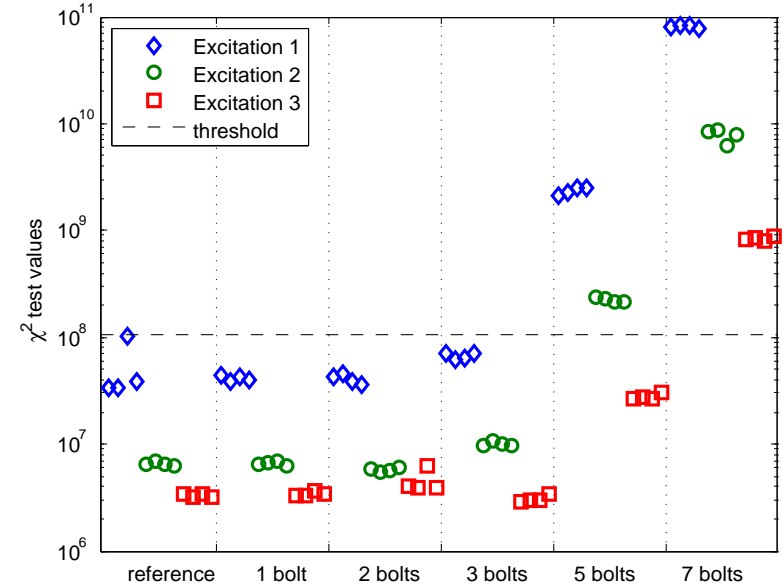

(a) Conventional test (10).

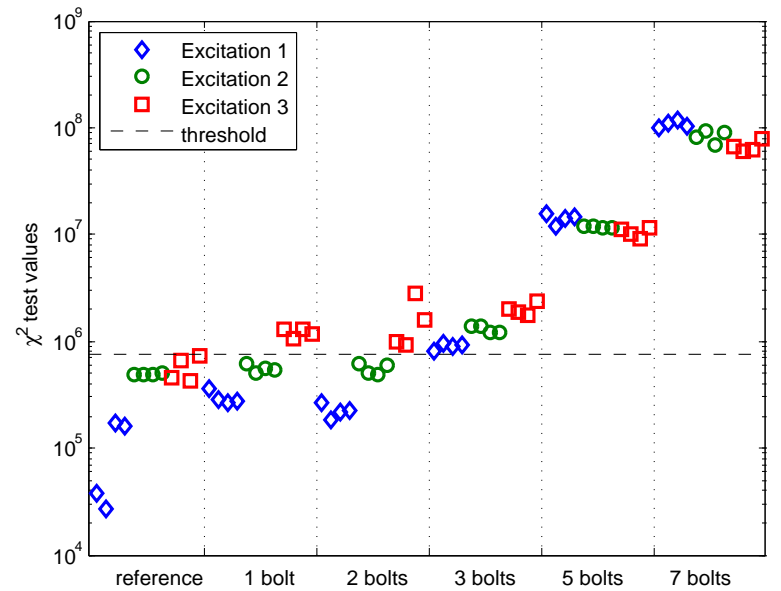

(b) New robust test (12).

Figure 4: Comparison of damage detection tests on steel frame structure in a lab experiment (log-scale).

\section{Conclusions}

In this paper, the recently developed statistical subspace-based damage detection test from [6] that is robust to changing ambient excitation properties was successfully tested on real data from a lab experiment for the first time. In this experiment a steel frame structure was artificially damaged by loosening bolts of a flange connection and output-only data was recorded under different excitation levels. The new test that was designed to be robust to changing excitation properties showed much better damage 
detection results in this setting with an earlier detection of damage than a previous conventional damage detection test. Since the applied damage detection framework already proved to be feasible for real civil structures in the field (see e.g. case studies $[9,14,4])$, the presented test robust to excitation changes should be of value for such applications, where the excitation is ambient and uncontrolled, and where the new test is thus expected to detect damage earlier and more reliably. The application of the new test to structures in the field is part of future work.

\section{Acknowledgments}

The partial support from the European project FP7-PEOPLE-2009-IAPP 251515 ISMS is gratefully acknowledged.

\section{References}

[1] E. Balmès, M. Basseville, F. Bourquin, L. Mevel, H. Nasser, and F. Treyssède. Merging sensor data from multiple temperature scenarios for vibration-based monitoring of civil structures. Structural Health Monitoring, 7(2):129-142, 2008.

[2] M. Basseville, M. Abdelghani, and A. Benveniste. Subspace-based fault detection algorithms for vibration monitoring. Automatica, 36(1):101-109, 2000.

[3] M. Basseville, L. Mevel, and M. Goursat. Statistical model-based damage detection and localization: subspace-based residuals and damage-to-noise sensitivity ratios. Journal of Sound and Vibration, 275(3):769-794, 2004.

[4] M. Döhler, F. Hille, L. Mevel, and W. Rücker. Structural health monitoring with statistical methods during progressive damage test of S101 Bridge. Engineering Structures. Under revision.

[5] M. Döhler and L. Mevel. Subspace-based fault detection robust to changes in the noise covariances. Automatica, 49(9):2734-2743, 2013.

[6] M. Döhler, L. Mevel, and F. Hille. Subspace-based damage detection under changes in the ambient excitation statistics. Mechanical Systems and Signal Processing, 45(1):207-224, 2014.

[7] M. Döhler, L. Mevel, and D. Siegert. Statistical subspace-based damage detection under changing ambient excitation. In Proc. 5th European Conference on Structural Control, Genoa, Italy, 2012.

[8] F. Magalhães, A. Cunha, and E. Caetano. Dynamic monitoring of a long span arch bridge. Engineering Structures, 30(11):3034-3044, 2008.

[9] L. Mevel, M. Goursat, and M. Basseville. Stochastic subspace-based structural identification and damage detection and localization - Application to the Z24 Bridge benchmark. Mechanical Systems and Signal Processing, 17(1):143-151, 2003.

[10] B. Peeters and G. De Roeck. Reference-based stochastic subspace identification for output-only modal analysis. Mechanical Systems and Signal Processing, 13(6):855-878, 1999.

[11] L. Ramos, L. Marques, P. Lourenço, G. De Roeck, A. Campos-Costa, and J. Roque. Monitoring historical masonry structures with operational modal analysis: Two case studies. Mechanical Systems and Signal Processing, 24(5):1291-1305, 2010. Special Issue: Operational Modal Analysis.

[12] J. Strout, M. Döhler, D. Bernal, and L. Mevel. Changes in the statistics of ambient excitations in the performance of two damage detection schemes. In Proc. 30th International Modal Analysis Conference, Jacksonville, FL, USA, 2012.

[13] P. Van Overschee and B. De Moor. Subspace Identification for Linear Systems: Theory, Implementation, Applications. Kluwer, 1996.

[14] W. Zhou, Z. Wu, and L. Mevel. Vibration-based damage detection to the composite tank filled with fluid. Structural Health Monitoring, 9(5):433-445, 2010. 